\title{
Give credit where due: authorship at $\mathrm{OE}$
}

Adam Wax

Editor-in-Chief

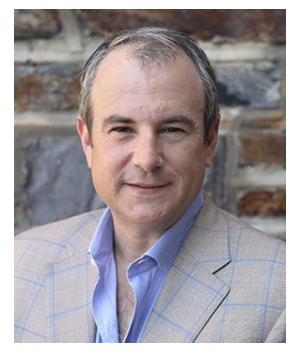

An important question that often comes up in academic publishing is whether to include someone as an author on your paper. The official policy of Optical Engineering is that authorship should be limited to those who have made a significant contribution to the article while those who do not meet this threshold should simply be acknowledged. This may seem to be somewhat of a gray area, but I believe the policy was drafted this way since it can be difficult for an editor to identify what qualifies as a significant contribution. Instead it is left up to the authors to decide.

As an academic writer myself, I have often used the following question to determine whether someone deserves authorship: Could this study have been completed without [this author's] contribution? If the answer is no, then it is clear that a significant contribution has been made. On the other hand, asking this question can make you realize that a contribution may have just been a technician completing routine lab duties or a colleague offering helpful suggestions. In that case, an author credit probably isn't warranted but rather just listed in the acknowledgments section.

Another tenet I have kept as an author is to err on the side of inclusiveness. That is, I would rather extend an author credit to a collaborator or lab member than to exclude them. This can avoid ill will and bad feelings among colleagues and co-workers. If you are going to intentionally leave someone off an author list, then an open conversation about his or her contribution to the study can help clear the air, particularly before the paper is submitted. Among newer academic authors, there can be a temptation to try to keep authorship lists to a minimum. The line of thinking is often borne out of competition or a fear that including more authors would "dilute" their own contribution. When I come across this type of situation, I try to use the opportunity to help encourage an approach that will foster collaboration. Authorship isn't a zero sum game, and it's always nicer to offer more seats at the table.

In contrast, a problem we have come across recently at $\mathrm{OE}$ is the proliferation of long author lists. In today's age of collaborative research, it is to be expected that there will be more authors per paper. However, it is not acceptable to gratuitously add authors who didn't contribute in a substantial way. For example, some authors feel adding a prestigious researcher may improve their chance of publication while others share authorship with friends to enhance publication lists and citations. This creates ethical issues for editors and reviewers when they see author lists of a dozen or more researchers contributing to a narrow technical advance. While some journals require a detailed list of author contributions to be included with submission, we do not require this at OE. However, editors can request a summary of contributions if they suspect that the author list has been padded.

To ensure that the author list for your paper is legitimate, take the time to verify that each author has contributed in the three phases of authorship: (1) contributed to an aspect of the design, execution, or data analysis of the study, (2) participated in the paper preparation, either with drafting or critical revision, and (3) provided approval of the final version. If an author meets these three criteria, then it's easy to justify that their contributions were indeed "significant."

() 2021 Society of Photo-Optical Instrumentation Engineers (SPIE) 